\section{Caution urged in trial of stem cells to treat spinal-cord injury}

SIR - As you point out in your News story 'Stem cells ready for prime time' (Nature 457, 516; 2009), the recent approval by the US Food and Drug Administration (FDA) of the first clinical trial to use derivatives of human embryonic stem (ES) cells is an important development. But the indication, namely spinalcord injury, raises concerns and important caveats.

First, the choice of a body area that is not simple to access is surprising in the context of what is meant to be primarily a safety trial. The field needs reliable information with regard to possible immune rejection of the transplanted cells and their potential to de-differentiate in situ after implantation.

Second, the benefits are not likely to be easy to assess, given what has been experienced with animal models of spinal-cord lesion. Difficulties in reproducing the lesion and in assessing both the damage and regrowth of axons after experimental manipulations have made results hard to interpret. It is generally agreed that cut axons cannot readily regrow in adult mammals, but it is not clear why. Damage caused by inflammatory cells, scar formation and decreased intrinsic growth drive of adult neurons, as well as the inhibitory effect of myelin in the central nervous system, may all play a part.

The trial approved by the FDA consists of injecting oligodendrocyte precursors into the spinal cord of patients soon after injury, implying that a lack of such precursors may be the key issue. Oligodendrocyte precursors do seem to myelinate axons after lesion in rodents $(\mathrm{H}$. S. Keirstead et al. J. Neurosci. 25, 4694-4705; 2005). However, the results still need to be interpreted in the context of what goes wrong after spinal-cord injury, including the possibility that axonal elongation may actually be impaired by oligodendrocyte-derived products.

Nonetheless, the generation of virtually pure populations of oligodendrocyte precursors is impressive, one of very few cases of cellular homogeneity of a differentiated cell type generated from ES cells (G. I. Nistor et al. Glia 49, 385-396; 2005). This achievement opens the way to studying the impact of mutations after reprogramming somatic cells from patients, and drug screening in a relevant cellular context.

Given the controversy over the use of human ES cells in some countries, it is to be hoped that the triumphant announcement of the approval of this clinical trial will not prove to be a prime-time setback. Regenerative medicine using human ES cells is an exciting prospect, but the field still needs time to mature. The primary concern of scientists involved in stem-cell research is not to satisfy the short-term expectations of analysts and investors, but to improve public health with the help of innovative, safe treatments. Yves Barde Biozentrum, University of Basel, Klingelbergstrasse 50-70, 4056 Basel, Switzerland e-mail:yves.barde@unibas.ch

\section{Cuts to research funding could hurt health care too}

SIR - The rapidly deepening financial crisis, reported on Nature's Recession Watch site (http://tinyurl.com/d4gy28), raises important questions about the future of medical research at a time when health care itself is under pressure.

As the crisis grows in pace and extent, it is difficult to predict its full effects, even on statebacked organizations such as the US National Institutes of Health (NIH) and the UK Medical Research Council (MRC) and National Health Service (NHS). Organizations such as the MRC and $\mathrm{NIH}$, which receive direct funding from governments, also work closely with research councils, industry and other stakeholders to produce highquality research. Active research aids decision-making about health standards, health policy and discovery of new drugs and treatments. There is evidence that doing research during medical training enhances a student's vision and performance later in academic research.

If funding to research organizations is reduced, the results may not be seen for three to five years - but they will be devastating. Global measures need to be taken to secure both domestic healthcare requirements and research funding.

First, it is imperative to continue to improve standards of health care and patient safety. Second, additional funds must be allocated to continue health care research while safeguarding basic health needs. Finally, alternatives for financing health care and research should be explored. An amalgamation between healthcare and research agendas could shield organizations from disastrous expenditure in this financially grim period.

Kamran Ahmed, Hutan Ashrafian Department of Biosurgery and Surgical Technology, Imperial College London, St Mary's Hospital Campus, London W2 1NY, UK e-mail:k.ahmed@imperial.ac.uk

\section{Separate name for fungus's sexual stage may cause confusion}

SIR - I applaud the discovery by C. M. O'Gorman and colleagues of a sexual stage in the medically important fungus Aspergillus fumigatus (Nature 457, 471-474; 2009). However, I question the introduction of a new scientific name for it, Neosartorya fumigata.

Mycologists know from morphological and now molecular phylogenetic evidence that the asexual $A$. fumigatus belongs with species that also produce a Neosartorya sexual stage. In the molecular era, the need for a single species to have separate scientific names for its various asexual stages has become obsolete. Species with no sexual stage can be placed with those that have. Even in the 1960s, Aspergillus monographer Kenneth Raper used one name for one fungus in Aspergillus.

Mycology is unique in permitting different binomials to be applied to the various sporing stages of the same living species. The need to evolve from this archaic position to that of a single name for one species in all its sporing states was advocated by Don Reynolds and John Taylor in 1991. In 2005, the XVII International Botanical Congress established a committee to consider options for changing the pertinent rules: that committee is due to report in 2011.

O'Gorman and colleagues did not contravene the current international rules in introducing $N$. fumigata, but have precipitated a potential source of confusion. That name is now available to apply to all stages of this fungus, including the asexual. The scientific community faces the prospect of reference to this species, even when dealing with just the asexual stage, using only the Neosartorya name.

The pragmatic approach is to use only the name $A$. fumigatus, regardless of whether the sexual or the asexual stage of the fungus is being referred to. This would be in line with the practice in A. nidulans, where the available name Emericella nidulans, based on the sexual stage of that species, is largely ignored.

David L. Hawksworth Facultad de Farmacia, Universidad Complutense de Madrid, Plaza Ramón y Cajal, Madrid 28040, Spain; and Department of Botany, Natural History Museum, Cromwell Road, London SW7 5BD, UK e-mail:d.hawksworth@nhm.ac.uk

Contributions may be submitted to correspondence@nature.com and will be edited. 